\title{
Matrix Metalloproteinases and Tissue Inhibitors of Matrix Metalloproteinases as Plasma Indicators of Bovine Cystic Ovarian Disease
}

\author{
K. F. Hentze, K. G. Younger, A. R. Menino* \\ 112 Withycombe Hall, Department of Animal and Rangeland Sciences, Oregon State University, Corvallis, Oregon, \\ USA
}

Correspondence to: A. R. Menino, alfred.r.menino@oregonstate.edu

Keywords: MMP, TIMP, Cattle, Ovulation

Received: April 26, $2018 \quad$ Accepted: August 25, $2018 \quad$ Published: August 28, 2018

Copyright @ 2018 by authors and Scientific Research Publishing Inc.

This work is licensed under the Creative Commons Attribution International License (CC BY 4.0).

http://creativecommons.org/licenses/by/4.0/

\section{(c) (i) Open Access}

\section{ABSTRACT}

Cystic ovarian disease (COD) is one of the main causes of infertility in dairy cattle and has a high economic impact on farmers. COD is caused by an endocrine imbalance within the hypothalamic-pituitary-ovarian axis preventing the mature Graafian follicle from ovulating. The cause at the molecular level is not well understood. However, the matrix metalloproteinases (MMP) and plasminogen activator/plasmin families of extracellular (ECM) matrix-degrading proteinases are involved in Graafian follicle breakdown and oocyte release in the ovulation process. Our research investigated the possibility of using plasma concentrations of MMP-2 and -9 and their natural tissue inhibitors (TIMP) -1 and -2 as prognostic indicators of COD. Plasma samples from cystic and non-cystic dairy cows were analyzed using ELISA. Although plasma concentrations of MMP-2 and -9 were greater and TIMP-2 was lower in non-cystic compared to cystic cows, no significant differences were observed in MMP-2 and -9 and TIMP-1 and -2 due to cyst status. However, the TIMP-1:MMP-9 and TIMP-2:MMP-2 molar ratios were greater, $(P=0.099)$ and $(P=0.038)$, respectively, in cystic compared to non-cystic cows, suggesting a proteolytic insufficiency in cows with COD that may be a contributing factor to the anovulatory pathology. These data may provide the groundwork for future research and development of tools for dairy farmers to selectively choose replacement heifers less likely to develop COD.

\section{INTRODUCTION}

Cystic ovarian disease (COD) is a reproductive pathology primarily affecting dairy cattle causing irregular estrous cycles and prolonging the postpartum interval to first estrus. Ovarian cysts are defined as 
ovarian structures over $2.5 \mathrm{~cm}$ which have persisted for longer than 10 days without a corpus luteum present [1]. There are two types of ovarian cysts: follicular and luteal. Follicular cysts are single or multiple thin-walled Graafian follicle structures whereas luteal cysts are generally single structures with a thicker wall of luteal tissue [2]. Of the two, follicular cysts account for approximately 70\% of COD cases [2]. Luteal cysts are often follicular cysts that have persisted and formed a thicker layer of luteal tissue around the edges of the cyst [3]. COD is associated with a dysfunction of the hypothalamic-pituitary-ovarian axis, but there is no consensus on a specific intra-ovarian dysfunction that causes COD [4]. Stangaferro et al. [5] found altered expression of components in the activin-inhibin-follistatin system. This system could be responsible for the endocrine alterations and follicular persistence seen in COD. Other factors can be stress, herd management, nutritional status, body condition, and infectious disease $[4,6]$.

Ovulation is dependent on breakdown of the ECM in the follicle wall by the MMP and plasminogen activator/plasmin families of proteinases. The MMP family consists of 26 different proteins, all of which are proteolytic enzymes. MMP-2 and -9 are in the gelatinase family of MMP and are involved in ECM remodeling during the estrous cycle [7, 8] and ovulation [9-11]. Differences in MMP-2 and -9 expression were reported by Imai et al. [12] where bovine follicular cysts had greater proMMP-2 and -9 activities in follicular fluid compared to follicles smaller than the threshold for cystic follicles. Follicular as well as plasma concentrations of MMP-2 and -9 and tissue inhibitor of MMP-1 (TIMP-1) were also higher in women with polycystic ovarian syndrome (PCOS), also an anovulatory condition, compared to healthy control women [13-15].

Failure of the mature Graafian follicle to ovulate in a timely fashion is a concern for dairymen because it decreases the reproductive efficiency of cows thereby increasing culling rates and costs of production. Over a lifetime, COD is estimated to affect $10 \%$ to $30 \%$ of high producing dairy cows [2]. If differences in plasma concentrations between normal and cystic cows correlate with follicular expression of MMP-2 and -9 and TIMP then plasma MMP could emerge as an indicator of COD in dairy cattle. This relationship could assist with providing dairy producers tools for selecting replacement heifers less likely to develop COD. The ramifications could be great for the dairy industry by providing a reduction in veterinary, breeding and culling costs. Therefore, the objective of this study was to quantify plasma concentrations of MMP-2 and -9 and TIMP-1 and -2 in cows diagnosed with COD and cows with no history of follicular cysts.

\section{MATERIALS AND METHODS}

\subsection{Animals}

A total of 65 lactating Holstein cows were used in this study and were housed at a cooperating dairy in Coburg, Oregon. This dairy had approximately 3000 cows with 1500 milking. Cows were provided with free-choice water and a total mixed ration consisting of corn silage, grass silage, alfalfa, corn, cotton seed and soy bean meal. Cows were divided into two groups based on ovarian palpation during herd health evaluations by a licensed veterinarian at 14-day intervals. Cows diagnosed with an ovarian follicular cyst, a follicle $>2.5 \mathrm{~cm}$ in diameter $[1,2]$, were assigned to the cyst group and a blood collection was performed. Cows selected to serve as the control, or non-cystic group, were cows observed in estrus the day of the blood collection by the herdsmen. Signs of estrus are standing to be mounted, increased step count and decreased milk production. For the non-cystic cows, medical records were inspected to verify no history of follicular cysts. Age and parity data were collected and for cystic cows, the number of lactations in which a cow had been diagnosed with a cyst was recorded. Power calculations were used to provide an estimate of sample size. All work was performed in accordance with the Oregon State University Institutional Animal Care and Use Committee.

\subsection{Blood Collection}

Blood samples were collected via coccygeal venipuncture using $10 \mathrm{~mL}$ Vacutainer (Becton Dickinson) 
blood-collection tubes. Vacutainers for plasma collection were preserved with heparin as the anticoagulant. Blood samples were transported back to the laboratory within approximately one hour of collection. Tubes were centrifuged at $5000 \mathrm{X}$ g for 10 minutes at $4^{\circ} \mathrm{C}$ to separate plasma from whole blood. Three $500-\mathrm{ml}$ aliquots of plasma were collected from the top half of each vacutainer, and each tube was labeled with "A", "B" or "C" according to the order in which the sample was taken, date of blood draw, and cow number. Aliquots were stored at $-20^{\circ} \mathrm{C}$ until analysis for MMP-2 and -9 and TIMP-1 and -2 .

\subsection{ELISA}

Plasma MMP-2 and -9 concentrations were quantified using Genorise (Berwyn, PA) ELISA kits. One-hundred microliters of standard or sample plasma in duplicate were incubated in a pre-coated antibody plate for one hour at RT. Any targeted MMP present in the sample were bound to the antibody on the plate. After the initial incubation, each well was washed with $200 \mu \mathrm{l}$ of wash buffer 4 times. One hundred microliters of detection antibody specific for bovine MMP-2 or -9 were added to each well in the plate and the plate was incubated for one hour at RT. Another wash was completed to remove unbound antibody reagent. One hundred microliters of detection reagent were added to each well and incubated for 20 minutes. Another wash cycle was completed and $100 \mu \mathrm{l}$ of substrate were added, which causes color formation during the 20-minute incubation at RT. Fifty microliters of stop solution were added to the wells to halt color development. The optical density (OD) of each well in the plate was immediately quantified at $450 \mathrm{~nm}$ and $550 \mathrm{~nm}$ using a BIOTEK EL800 plate reader.

To calculate MMP-2 and -9 plasma concentrations in the samples, OD measurements at $550 \mathrm{~nm}$ were subtracted from OD at $450 \mathrm{~nm}$ as a correction factor for imperfections in the plate. Corrected OD measurements were transformed into plasma concentrations using Excel and equation of the line calculations. A standard curve was created using the OD readings of the standards provided in the Genorise ELISA kit. Plasma concentrations of MMP-2 and -9 in the samples were determined by entering OD readings into the standard curve equation.

TIMP-1 concentrations were quantified using a MyBioSource (San Diego, CA) ELISA kit. All reagents were brought to RT prior to starting the assay. One hundred microliters of standard or sample were added to each well in the assay plate. All standards were run in duplicate. A closure membrane was then placed on top of the plate while the tray incubated for two hours at $37^{\circ} \mathrm{C}$. After the first incubation, all liquid was removed from the wells and $100 \mu \mathrm{l}$ of detection reagent $\mathrm{A}$ were added to each well. The plate was covered again and incubated for one hour at $37^{\circ} \mathrm{C}$. Each well was aspirated and washed four times using $400 \mu \mathrm{l}$ of wash buffer. One hundred microliters of detection reagent $\mathrm{B}$ were added to each well and the plate was covered again for 1 hour at $37^{\circ} \mathrm{C}$. Each well was washed five times. After all liquid was removed from the wash steps, $90 \mu \mathrm{l}$ of substrate solution were added to each well. The plate was sealed and incubated at $37^{\circ} \mathrm{C}$ for 15 minutes, while being protected from light. Fifty microliters of stop solution were added to each well and the OD of each well was quantified within 5 minutes using a BIOTEK EL800 plate reader. Blank wells served as the correction factor.

TIMP-2 concentrations were also quantified using a MyBioSource (San Diego, CA) ELISA kit. All samples and reagents were brought to RT 30 minutes before starting assay procedures. Fifty microliters of sample, standard or sample diluent were added to each well in the assay plate. All standards were run in duplicate. Sample diluent was used as a blank control sample in duplicate. One hundred microliters of HRP-conjugate were added to each well. A closure membrane was placed on top of the plate and the tray was incubated at $37^{\circ} \mathrm{C}$ for 60 minutes. The plate was washed with approximately $400 \mu \mathrm{l}$ of wash buffer four times. Fifty microliters of Chromogen solution A and B were added successively to each well. The tray was protected from light, covered with a membrane, and incubated at $37^{\circ} \mathrm{C}$ for 15 minutes. Fifty microliters of stop solution were added to each well and OD was measured at $450 \mathrm{~nm}$ within 15 minutes of adding the stop solution in a BIOTEK EL800 plate reader. Blank wells served as the correction factor.

To calculate plasma TIMP-1 and -2 concentrations in the samples, the average OD of the blank wells were subtracted from the OD of each standard as a correction factor. Corrected OD measurements were 
transformed into plasma concentrations using Excel and equation of the line calculations. A standard curve was created using the OD readings of the standards provided in the MyBioSource ELISA kit. Plasma concentrations of TIMP-1 and -2 in the samples were determined by entering corrected OD readings into the standard curve equation.

\subsection{Statistical Analyses}

Differences in plasma concentrations of MMP-2 and -9, TIMP-1 and -2 and the TIMP-1:MMP-9 and TIMP-2:MMP-2 molar ratios due to cyst status (cystic vs. non-cystic), cyst number, parity and age were determined by one-way ANOVA. If significant effects were observed in the ANOVA, differences between means were evaluated by Fisher's least significant differences procedures. Correlation-regression analyses were conducted to determine the degree of relationship in the TIMP-1:MMP-9 and TIMP-2:MMP-2 molar ratios with age and parity. All analyses were performed using the NCSS statistical software program (Number Cruncher Statistical System; 2007, Jerry Hintze, Kaysville, UT).

\section{RESULTS}

For MMP-2 and -9 quantification, each group, cystic and non-cystic, consisted of 32 cows. For TIMP-1 and -2, 33 cystic and 32 non-cystic cows were sampled. Average ages and parities of cystic and non-cystic cows were $44.4 \pm 3.3$ and $37.8 \pm 2.2$ months and $2.0 \pm 0.3$ and $1.8 \pm 0.2$ parities, respectively, and did not differ $(\mathrm{P}>0.10)$ between groups.

\subsection{MMP-2 and -9}

Although MMP-2 concentrations were greater in plasma recovered from non-cystic compared to cystic cows, no difference was observed $(\mathrm{P}=0.33)$. Mean plasma concentrations of MMP-2 in cystic and non-cystic cows were $228.8 \pm 49.8$ and $311.5 \pm 68.2 \mathrm{pg} / \mathrm{ml}$, respectively. Intra-assay coefficient of variation for MMP-2 assays was $15.4 \%$.

Similar to MMP-2, MMP-9 concentrations were greater in plasma recovered from non-cystic compared to cystic cows, however, no difference was observed $(\mathrm{P}=0.76)$. Mean plasma concentrations of MMP-9 in cystic and non-cystic cows were $89.5 \pm 14.6$ and $95.4 \pm 12.3 \mathrm{pg} / \mathrm{ml}$, respectively. Intra-assay coefficient of variation for MMP-9 assays 1 and 2 was $7.5 \%$.

\subsection{TIMP-1 and -2}

No difference $(\mathrm{P}=0.86)$ in plasma TIMP-1 concentrations was detected between cows diagnosed with a cystic follicle and normal cows with no history of COD. Mean plasma concentrations of TIMP-1 in cystic and non-cystic cows were $4.71 \pm 0.8$ and $4.96 \pm 1.2 \mathrm{ng} / \mathrm{ml}$. Intra-assay coefficient of variation for the TIMP-1 assay was $9.0 \%$.

Although TIMP-2 concentrations were lower in plasma recovered from non-cystic cows compared to cystic cows, no difference was observed $(\mathrm{P}=0.15)$. Mean plasma concentrations of TIMP-2 in cystic and non-cystic cows were $39.7 \pm 1.4$ and $36.9 \pm 1.3 \mathrm{ng} / \mathrm{ml}$. Intra-assay coefficient of variation for the TIMP-2 assay was $3.7 \%$.

\subsection{MMP-2 and -9 Concentrations Relative to Cyst Number}

MMP-2 and -9 plasma concentrations were analyzed relative to the number of lactations a cow had been diagnosed with a cyst. MMP-2 plasma concentrations decreased as number of cysts increased, except in the 3 cyst group, however no differences $(\mathrm{P}=0.79)$ were observed (Figure 1$)$. MMP-9 plasma concentrations remained similar $(\mathrm{P}=0.98)$ in all groups (Figure 2$)$.

\subsection{TIMP-1 and -2 Concentrations Relative to Cyst Number}

Likewise, TIMP-1 and -2 plasma concentrations were analyzed relative to the number of lactations a 


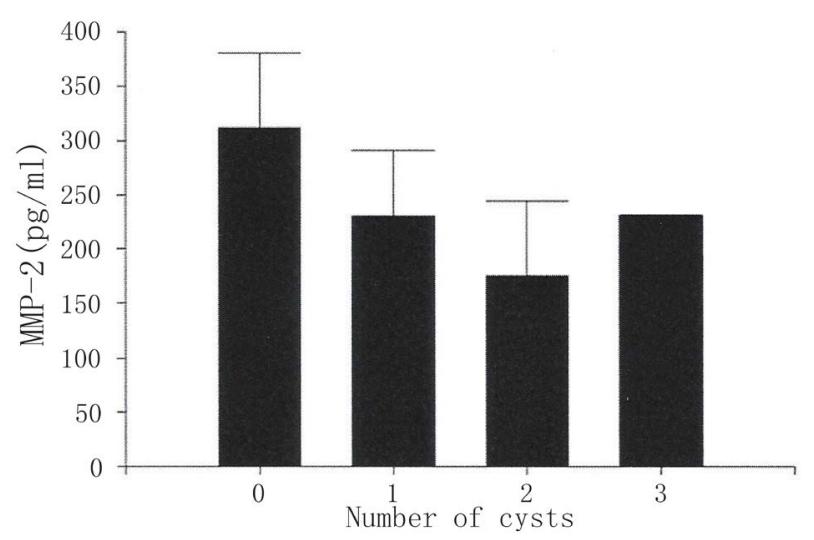

Figure 1. MMP-2 plasma concentrations (mean \pm $\mathrm{SE})$ in cows with $0(\mathrm{n}=31), 1(\mathrm{n}=26), 2(\mathrm{n}=3)$ or 3 cysts $(n=1)$.

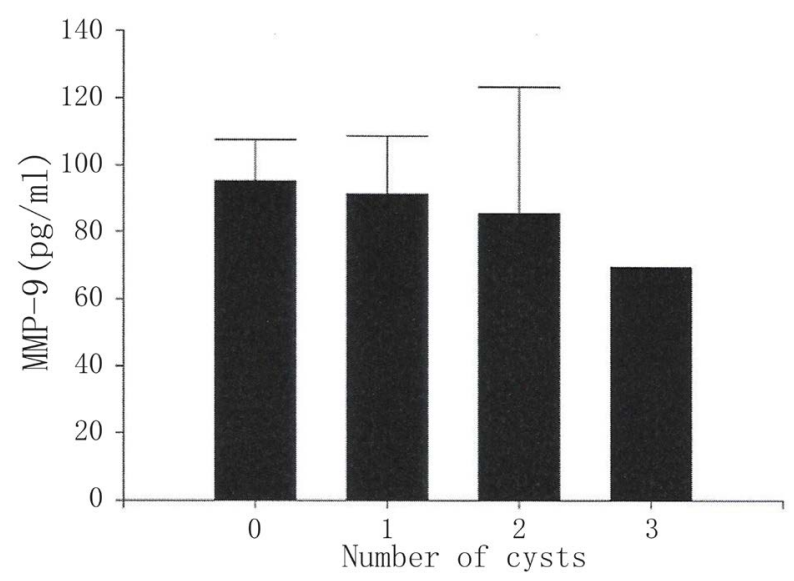

Figure 2. MMP-9 plasma concentrations (mean \pm $\mathrm{SE})$ in cows with $0(\mathrm{n}=31), 1(\mathrm{n}=26), 2(\mathrm{n}=3)$ or 3 cysts $(n=1)$.

cow had been diagnosed with a cyst. Although TIMP-1 plasma concentrations decreased as number of cysts increased, no differences $(P=0.90)$ were observed (Figure 3$)$. No differences $(P=0.46)$ were observed due to cyst number in plasma concentrations of TIMP-2, however the cow with a history of 3 cysts had the highest plasma concentration (Figure 4).

\subsection{TIMP-1:MMP-9 and TIMP-2:MMP-2 Molar Ratios}

TIMP-1:MMP-9 molar ratio was greater $(\mathrm{P}=0.099)$ in cystic compared to non-cystic cows (Figure 5). Similarly, TIMP-2:MMP-2 molar ratio was greater $(\mathrm{P}=0.038)$ in cystic compared to non-cystic cows (Figure 6). Cystic cows with one cyst in their production record had a TIMP-1:MMP-9 molar ratio twofold greater $(\mathrm{P}<0.05)$ than non-cystic cows (Figure 7). The TIMP-2:MMP-2 molar ratio was four times $(\mathrm{P}$ $<0.05$ ) greater in cystic cows with one cyst compared to non-cystic cows (Figure 7).

Correlations for TIMP-1:MMP-9 and TIMP-2:MMP-2 molar ratios with age and parity were negative for cystic cows and only the TIMP-1:MMP-9 molar ratio was meaningful ( $\mathrm{P}=0.06$; Table 1$)$. No significant correlations were observed for TIMP-1:MMP-9 and TIMP-2:MMP-2 molar ratios with age and parity for non-cystic cows (Table 2). 


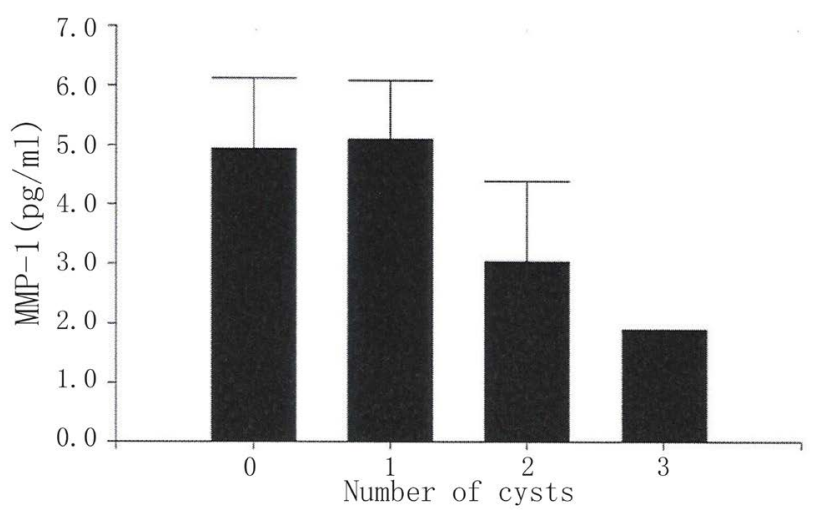

Figure 3. TIMP-1 plasma concentrations (mean \pm $\mathrm{SE})$ in cows with $0(\mathrm{n}=32), 1(\mathrm{n}=26), 2(\mathrm{n}=3)$ or 3 cysts $(n=1)$.

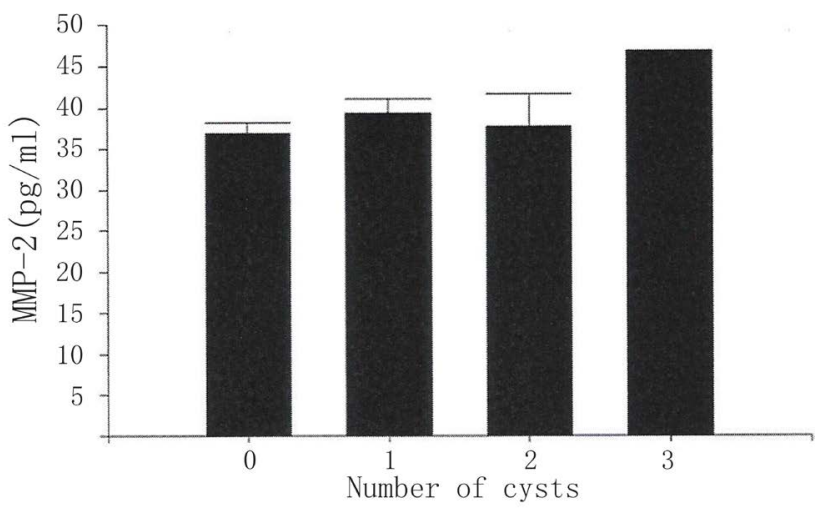

Figure 4. TIMP-2 plasma concentrations (mean \pm $\mathrm{SE})$ in cows with $0(\mathrm{n}=32), 1(\mathrm{n}=26), 2(\mathrm{n}=3)$ or 3 cysts $(n=1)$.

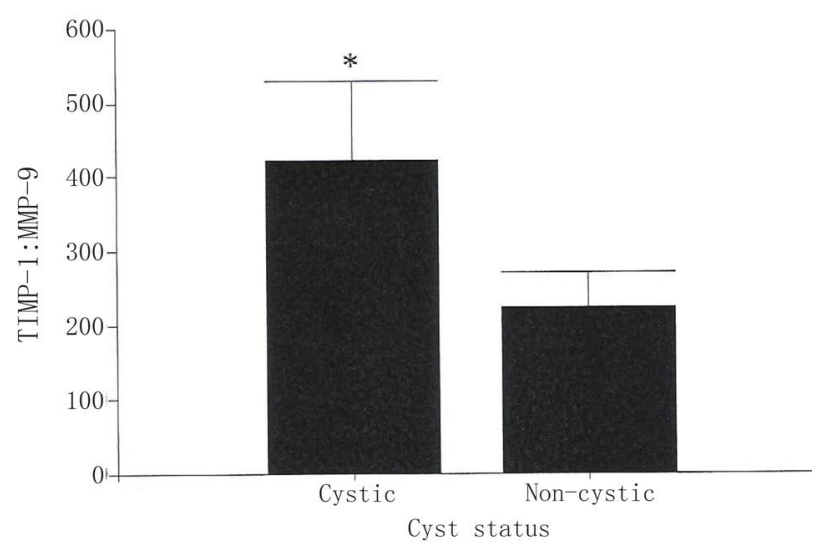

Figure 5. Molar ratios of TIMP-1:MMP-9 (mean \pm $\mathrm{SE})$ in cows diagnosed with a follicular cyst $(\mathrm{n}=$ 31) or non-cystic cows $(n=31)$. ${ }^{\star}$ Different from non-cystic cows $(P=0.099)$. 


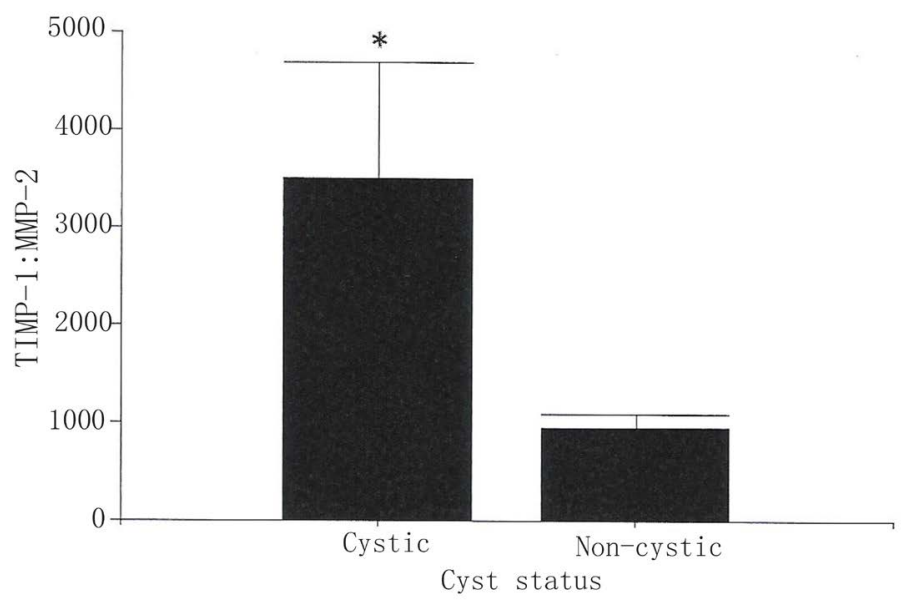

Figure 6. Molar ratios of TIMP-2:MMP-2 (mean $\pm \mathrm{SE}$ ) in cows diagnosed with a follicular cyst $(n=32)$ or non-cystic cows $(\mathrm{n}=31)$. ${ }^{*}$ Different from non-cystic cows $(P=0.038)$.

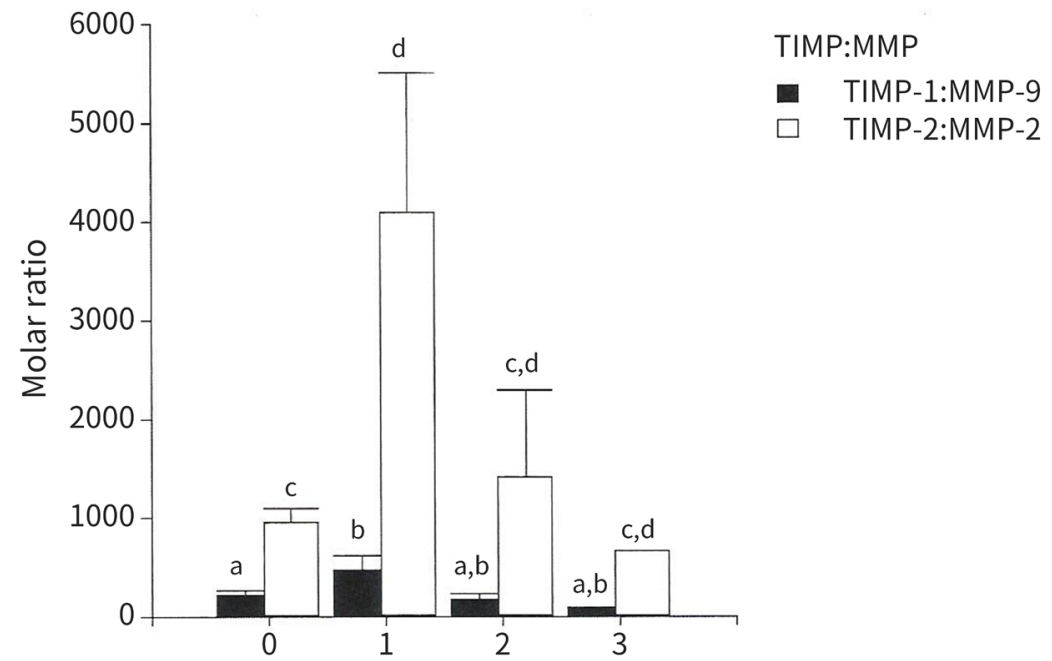

Figure 7. Molar ratios of TIMP-1:MMP-9 (mean \pm SE) in cows diagnosed with $0(n=31), 1(n=25), 2(n=3)$ or 3 cysts $(n=$ 1) and TIMP-2:MMP-2 (mean \pm SE) in cows diagnosed with 0 $(\mathrm{n}=31), 1(\mathrm{n}=26), 2(\mathrm{n}=3)$ or 3 cysts $(\mathrm{n}=1) \cdot{ }^{\mathrm{a}, \mathrm{b}}$ Means without common superscripts for TIMP-1:MMP-9 molar ratios differ $(P<0.05)$; ${ }^{c, d}$ Means without common superscripts for TIMP-2:MMP-2 molar ratios differ $(P<0.05)$.

\section{DISCUSSION}

These data suggest cows diagnosed with COD do not have significantly altered plasma concentrations of MMP-2, MMP-9, TIMP-1 or TIMP-2 compared to cows with no history of cystic follicles. Although no statistical differences in plasma MMP-2 and -9 concentrations were observed between normal and cystic cows, both MMP-2 and -9 were higher in cows with no cystic history compared to cystic cows. In fact, 
Table 1. Correlation coefficients (r) for TIMP-1:MMP-9 and TIMP-2:MMP-2 molar ratios by parity and age in cystic cows.

\begin{tabular}{ccccc}
\hline Y-variable & X-variable & $\mathbf{n}$ & $\mathbf{r}$ & P-value \\
\hline TIMP-1:MMP-9 & Age (mons) & 29 & -0.353 & 0.06 \\
TIMP-1:MMP-9 & Parity & 29 & -0.293 & 0.12 \\
TIMP-2:MMP-2 & Age (mons) & 30 & -0.102 & 0.59 \\
TIMP-2:MMP-2 & Parity & 30 & -0.141 & 0.46
\end{tabular}

Table 2. Correlation coefficients ( $r$ ) for TIMP-1:MMP-9 and TIMP-2:MMP-2 molar ratios by age and parity in non-cystic cows.

\begin{tabular}{ccccc}
\hline Y-variable & X-variable & $\mathbf{n}$ & $\mathbf{r}$ & P-value \\
\hline TIMP-1:MMP-9 & Age (mons) & 30 & -0.036 & 0.85 \\
TIMP-1:MMP-9 & Parity & 30 & -0.028 & 0.88 \\
TIMP-2:MMP-2 & Age (mons) & 30 & 0.017 & 0.93 \\
TIMP-2:MMP-2 & Parity & 30 & -0.026 & 0.89 \\
\hline
\end{tabular}

MMP-2 plasma concentrations were approximately 36\% higher in cows with no history of follicular cysts. However, noticeable between animal variation was observed in MMP-2 plasma concentrations, as evidenced by the SE associated with the means. Perhaps with a larger sample size significant differences may have been realized in MMP-2. Results from the present study differ from those of Imai et al. [12] who observed more proMMP-9 activity, albeit in follicular fluid, in bovine cystic follicles compared to follicles below the threshold diameter for cysts. Our results also differ from several studies reporting elevated concentrations of MMP-2 and -9 in plasma and follicular fluid in women with PCOS [13-15].

Concentrations of TIMP-1 were very similar in both groups and as TIMP-1 is often regarded as being constitutively expressed this observation was not surprising. TIMP-2 plasma concentrations were elevated in cystic compared to non-cystic cows and the difference approached significance with a P-value of 0.15 . TIMP-2 is tightly tied to regulation of MMP-2 and MMP-9 is regulated by multiple TIMP. Interestingly, serum TIMP-1, but not TIMP-2, was higher in women with PCOS compared to healthy women [14]. However Baka et al. [15] reported higher concentrations of both TIMP-1 and -2 in follicular fluid recovered from women with PCOS compared to women without the pathology.

Molar ratios of TIMP-1:MMP-9 and TIMP-2:MMP-2 were at least 100 and 1000-fold, respectively, greater in favor of TIMP compared to MMP. Both TIMP-1:MMP-9 and especially TIMP-2:MMP-2 molar ratios were greater in cystic cows compared to non-cystic cows suggesting an imbalance in the protease inhibitor to protease ratio in favor of reduced proteolysis in cows with COD. Whether this difference translates to impaired proteolysis at the follicular level is not known however it suggests a plausible explanation for the follicular cyst pathology where reduced follicular wall proteolysis could lead to the anovulatory condition.

Age and parity have been suggested to be factors associated with COD $[4,16]$. In the current study the TIMP-1:MMP-9 molar ratio approached significance for cystic cows where as age and parity increased the ratio decreased. However relationships with the TIMP-2:MMP-2 molar ratio and age and parity were decidedly nonsignificant. Meaningful relationships with either molar ratio with age and parity for non-cystic cows were not observed.

Multiple ECM degrading proteinase systems play a role in the regulation of ovulation. Having an imbalance in one of these multiple systems could play a role in COD. In the present study, molar ratios of TIMP-1:MMP-9 and TIMP-2:MMP-2 in plasma were greater in cystic cows compared to non-cystic cows 
and favored reduced proteolysis in cows with COD. If this difference translates to the ovarian level the impaired proteolysis may predicate development of the follicular cyst pathology. Whether the plasma TIMP-2:MMP-2 molar ratio can be used as a marker for heifers with a predilection to develop COD remains to be determined. However, given the economic losses suffered by dairy producers due to COD, evaluation of such a relationship bears merit for future research.

\section{CONCLUSION}

Molar ratios of TIMP-1:MMP-9 and TIMP-2:MMP-2 in plasma were greater in cows diagnosed with COD compared to non-cystic cows. This relationship could be used as a tool for dairy producers in selecting replacement heifers less likely to develop COD thereby increasing production efficiency by decreasing veterinary, breeding and culling costs.

\section{FINANCIAL DISCLOSURE}

The authors have no financial relationships relevant to this to disclose.

\section{CONFLICTS OF INTEREST}

The authors have no conflicts of interest to disclose.

\section{REFERENCES}

1. Roberts, S.J. (1955) Clinical Observations on Cystic Ovaries in Dairy Cattle. The Cornell Veterinarian, 45, 497-505.

2. Kesler, D.J. and Garverick, H.A. (1982) Ovarian Cysts in Dairy Cattle: A Review. Journal of Animal Science, 55, 1147-1159. https://doi.org/10.2527/jas1982.5551147x

3. Garverick, H.A. (1997) Ovarian Cysts in Dairy Cows. Journal of Dairy Science, 80, 995-1004. https://doi.org/10.3168/jds.S0022-0302(97)76025-9

4. Silvia, W.J., Hatler, T.B, Nugent, A.M. and Laranja da Fonseca, L.F. (2002) Ovarian Follicular Cysts in Dairy Cows: An Abnormality in Folliculogenesis. Domestic Animal Endocrinology, 23, 167-177. https://doi.org/10.1016/S0739-7240(02)00154-6

5. Stangaferro, M., Matiller, V., Diaz, P., Ortega, H., Rey, F., Rodriguez, F., Silvia, M. and Salvetti, N. (2014) Role of Activin, Inhibin, and Follistatin in the Pathogenesis of Bovine Cystic Ovarian Disease. Animal Reproduction Science, 148, 97-108. https://doi.org/10.1016/j.anireprosci.2014.06.005

6. Casida, L.E. and Chapman, A.B. (1951) Factors Affecting the Incidence of Cystic Ovaries in a Herd of Holstein Cows. Journal of Dairy Science, 34, 1200-1205. https://doi.org/10.3168/jds.S0022-0302(51)91848-6

7. Matthew, J.G., Marsha, A.M. and Paul, C.W. (1995) Identification of Matrix Metalloproteinases and Metalloproteinase Inhibitors in Bovine Corpora Lutea and Their Variation during the Estrous Cycle. Journal of Animal Science, 74, 849-857.

8. Curry, T.E. and Osteen, K.G. (2003) The Matrix Metalloproteinase System: Changes, Regulation, and Impact throughout the Ovarian and Uterine Reproductive Cycle. Endocrine Reviews, 24, 428-465. https://doi.org/10.1210/er.2002-0005

9. McIntush, E.W. and Smith, M.F. (1998) Matrix Metalloproteinases and Tissue Inhibitors of Metalloproteinases in Ovarian Function. Reviews of Reproduction, 3, 23-30. https://doi.org/10.1530/ror.0.0030023

10. Smith, M.F., McIntush, E.W., Ricke, W.A., Kojima, F.N. and Smith, G.W. (1999) Regulation of Ovarian Extracellular Matrix Remodeling by Metalloproteinases and Their Tissue Inhibitors: Effects on Follicular Development, Ovulation and Luteal Function. Journal of Reproduction and Fertility, 54, 367-381. 
11. Ny, T., Wahlberg, P. and Brändström, I. (2002) Matrix Remodeling in the Ovary: Regulation and Functional Role of the Plasminogen Activator and Matrix Metalloproteinase Systems. Molecular and Cellular Endocrinology, 187, 29-38. https://doi.org/10.1016/S0303-7207(01)00711-0

12. Imai, K., Khandoker, M., Yonai, M., Takahashi, T., Sato, T., Ito, A., Hasegawa, Y. and Hashizume, K. (2003) Matrix Metalloproteinases-2 and -9 Activities in Bovine Follicular Fluid of Different-Sized Follicles: Relationship to Intra-Follicular Inhibin and Steroid Concentrations. Domestic Animal Endocrinology, 24, 171-183. https://doi.org/10.1016/S0739-7240(02)00235-7

13. Shalev, E., Goldman, S. and Ben-Shlomo, I. (2001) The Balance between MMP-9 and MMP-2 and Their Tissue Inhibitor (TIMP)-1 in Luteinized Granulosa Cells: Comparison between Women with PCOS and Normal Ovulatory Women. Molecular Human Reproduction, 7, 325-331. https://doi.org/10.1093/molehr/7.4.325

14. Lewandowski, K.C., Komorowski, J., O’Callaghan, C.J., Tan, B.K., Chen J., Prelevic, G.M. and Randeva, H.S. (2006) Increased Circulating Levels of Matrix Metalloproteinase-2 and -9 in Women with the Polycystic Ovary Syndrome. Journal of Clinical Endocrinology and Metabolism, 91, 1173-1177. https://doi.org/10.1210/jc.2005-0648

15. Baka, S., Zourla, K., Kouskouni, E., Makrakis, E., Demeridou, S., Tzanakaki, D., Hassiakos, D. and Creatsas, G. (2010) Matrix Metalloproteinases 2 and 9 and Their Tissue Inhibitors in the Follicular Fluid of Patients with Polycystic Ovaries Undergoing in Vitro Fertilisation. In Vivo, 24, 293-296.

16. Peter, A.T. (1997) Infertility Due to Abnormalities of the Ovaries. In: Youngquist, R.S., Ed., Current Therapy in Large Animal Theriogenology, W.B. Saunders Co., Philadelphia, 349-354. 